\title{
Costal cartilage graft in Asian rhinoplasty: surgical techniques
}

\author{
Sarina Rajbhandari', Chuan-Hsiang Kao ${ }^{2}$ \\ 1Department of Otolaryngology - Head and Neck Surgery, Himal Hospital, Kathmandu 44600, Nepal. \\ ${ }^{2}$ Department of Otolaryngology - Head and Neck Surgery, Tri-Service General Hospital, Taipei 11490, Taiwan.
}

Correspondence to: Dr. Chuan-Hsiang Kao, Department of Otolaryngology-Head and Neck Surgery, Tri-Service General Hospital, No 325, Sec. 2nd, Cheng-Kung Road, Neihu District, Taipei 11490, Taiwan. E-mail: chuanhsiang@yahoo.com.tw

How to cite this article: Rajbhandari S, Kao CH. Costal cartilage graft in Asian rhinoplasty: surgical techniques. Plast Aesthet Res 2019;6:8. http://dx.doi.org/10.20517/2347-9264.2018.86

Received: 31 Dec 2018 First Decision: 7 Mar 2019 Revised: 19 Mar 2019 Accepted: 2 Apr 2019 Published: 18 Apr 2019

Science Editor: Hong Ryul Jin Copy Editor: Cai-Hong Wang Production Editor: Huan-Liang Wu

\begin{abstract}
Asian rhinoplasty is a very common cosmetic procedure. Many Asians desire a higher nasal bridge, for which they undergo several procedures, including filler injections, implantations and insertion of threads. Surgeons encounter many patients who have had several procedures done on them previously. In this paper, we introduce the use of autologous grafts for Asian rhinoplasty (primary and secondary), and discuss the rib carving techniques and difficulties encountered during harvesting, carving and placements of grafts and how to overcome these problems and prevent complications.
\end{abstract}

Keywords: Rhinoplasty, costal cartilage, Asians, warping

\section{INTRODUCTION}

Surgeons who perform Asian rhinoplasty often have to treat patients who have had rhinoplasty (or several rhinoplasties) previously. Revision rhinoplasty is one of the most difficult and challenging surgeries in facial plastic surgery.

For treating complications in rhinoplasty, a considerable quantity of cartilage is required to correct both nasal contour deformities and functional problems caused by previous surgeries. Revision Asian rhinoplasty tends to be a more complicated procedure than primary rhinoplasty, especially due to framework deficiency, that needs further reconstruction. For consistent long-term results, surgeons should use grafts with low

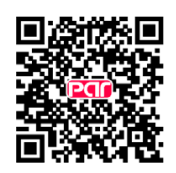


resorption rates and sufficient strength for framework support. Autologous tissue is always preferred as the use of alloplastic material increases the rate of infection, wound contracture and extrusion ${ }^{[1]}$.

For achieving successful results in Asian rhinoplasty, an appreciation of the Asian patient's anatomical characteristics, a conceptual approach as well as an appreciation of recent trends of beauty should be fully understood $^{[2]}$. Asian patients generally seek a high dorsum and nasal tip refinement. Silastic implants were used traditionally (and are still in use), but they cause a high incidence of early and late complications. Minimally invasive rhinoplasty, such as threads insertion rhinoplasty and injectable filler rhinoplasty have recently become popular, but repeated procedures may result in complications.

The ideal material for grafting or implantation in rhinoplasty must have the characteristics of low complication rates and high long-term patient satisfaction ${ }^{[3]}$. Thus, autografts are considered as better alternatives for augmentation in Asian rhinoplasties. Costal cartilage is commonly used for augmentation of the nasal dorsum and for infrastructure reconstruction as it provides an ample amount of autogenous cartilage, but is frequently associated with warping ${ }^{[4]}$. When an autologous rib cartilage rhinoplasty is performed properly by an experienced surgeon for complicated cases or for a short nose, it will provide excellent, reliable, and longlasting results with low risk ${ }^{[5]}$. Warping rate of costal cartilage and unpredictable cosmetic results are topics of concern for both patients and surgeons. In this paper, we will discuss how to minimize complications and improve the surgical results.

\section{SURGICAL TECHNIQUE}

\section{Harvesting the rib cartilage}

We usually harvest the rib cartilage from the right sixth or seventh rib, with a short linear inframammary incision [Figure 1A]. In women, we place an oblique incision carefully on the inframammary fold, to conceal the scar. The incision is around $2-3 \mathrm{~cm}$ in length. If a female patient wishes to opt for a breast implant in the future, we make the inframammary fold incision 7.5-8.0 cm below the nipple, so that the scar is hidden within the anticipated, future inframammary fold after breast implantation. If the patient has had a previous breast implant, the incision is made a little lower and we are careful not to rupture the capsule and prevent chances of breast implant infection.

We make an incision with a No. 10 or 15 blade and perform meticulous dissection of the subcutaneous tissue after infiltration of local anesthesia. Once we reach and divide the muscle fascia, the extra-costal muscle is divided directly over the rib. We identify the underlying rib and a syringe needle is stabbed on the costal cartilage to check for calcification. The medial dissection is near the junction of the rib cartilage and the sternum, while the lateral dissection is up to the osteochondral junction. We further carry out subperichondrium dissection underneath, along the longitudinal axis of the rib. Since we also aim to harvest some amount of perichondrium from the superior aspect of the rib, we make a rectangular incision on its superior aspect. Dissection is carried out carefully, with patience and accuracy, to leave the perichondrium on the lower aspect intact. We often use a curved or a right angled elevator to lift the rib off its underlying perichondrium. A blade is used to make an incision halfway through the rib and the costal cartilage is severed laterally near the osteo-chondral junction. The harvested rib measures $4.5-6.5 \mathrm{~cm}$ in length [Figure 1B]; we tend to harvest more costal cartilage in revision cases. The perichondrium on the superior aspect of the harvested rib [Figure 2] is preserved and kept aside. A sharp, curved Freer's elevator is used to make an incision at the medial end and sever the rib. A drill may be used to cut through the rib cartilage in cases of ossification, which is often seen in individuals over 40 years of age. Hence, we ask patients older than 40 years old to have a CT scan of the chest wall, for evaluation of costal cartilage calcification. But in our experience, rib calcification in younger individuals has also been noticed. In some patients, where there is 

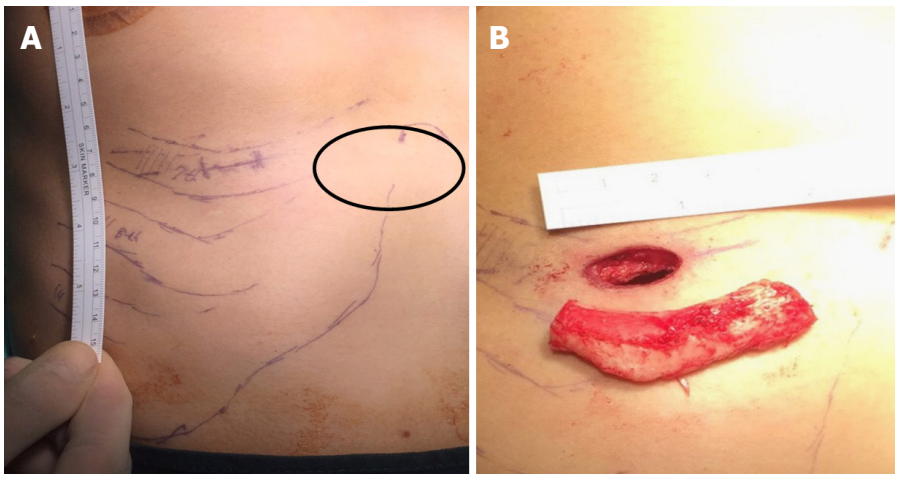

Figure 1. A: Incision site for harvesting the seventh rib; B: an approximately $5 \mathrm{~cm}$ rib harvested from a $2 \mathrm{~cm}$ incision

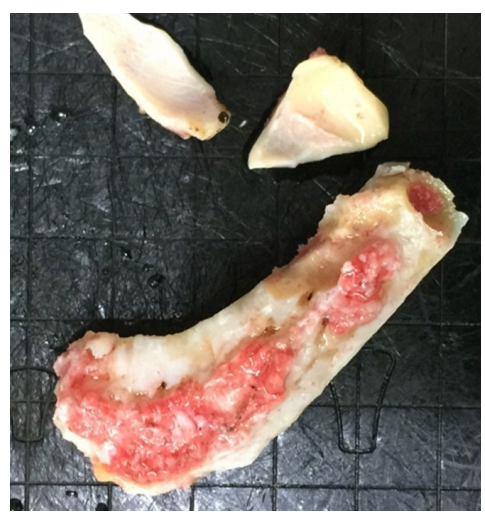

Figure 2. Harvested 7th rib cartilage with intact overlying perichondrium and two pieces of conchal cartilage

a considerable amount of calcification and if we suspect deficient septal cartilage, a short segment of the adjacent rib is also harvested, to use as splinting grafts or as a caudal septal extension graft.

The next crucial step is performing a Valsalva maneuver to check for injury to the lung pleura. The donor site is irrigated with thermal saline and positive pressure ventilation is applied to see the presence of bubbles. After ensuring that there is no pneumothorax, the wound is carefully closed in layers. The most important step is proper closure of fascia over the muscle with interrupted sutures. This will facilitate drainage of blood and avoidance of hematoma. Proper closure of this layer will also reduce postoperative pain over the chest area by a great extent. We generally use a 3-0 vicryl suture for closure of fascia over the muscle, 4-0 vicryl for subcutaneous closure and 6-0 nylon for interrupted closure of the skin. In female patients, we prefer to use 5-O PDS or vicryl suture for subcutaneous closure instead of 6-O nylon for skin closure; to avoid suture removal [Figure 3]. We then apply dressing over the wound to keep it dry and clean. The graft is put in normal saline with gentamycin solution and observed for warping.

\section{Carving the rib cartilage}

Carving and smoothening of the rib cartilage is crucial to get an even and aesthetically favorable outcome. Whilst carving a graft, we consider the patient's skin thickness. Asians have a thick skin, compared to that of Caucasians. This quality of skin comes with both an advantage and disadvantage. Minor irregularities on the nose may not be as obvious as it would be in the thinner Caucasian skin. But, for a patient requiring minimal changes, the final outcome may not be apparent and the patient might not be completely satisfied. It is difficult to achieve the desired level of definition and refinement because sharp lines and angles of the graft can appear blunted under a thick skin. 

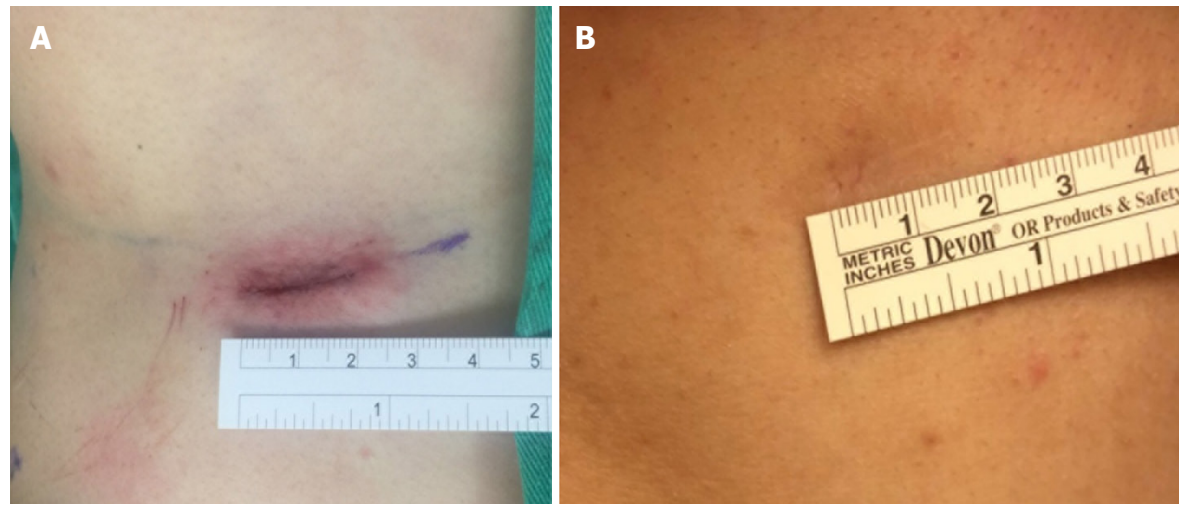

Figure 3. A: Oblique linear sutured wound about $2 \mathrm{~cm}$ over right inframammary fold after rib cartilage harvesting; B: a linear scar, appearing almost invisible, one year after surgery
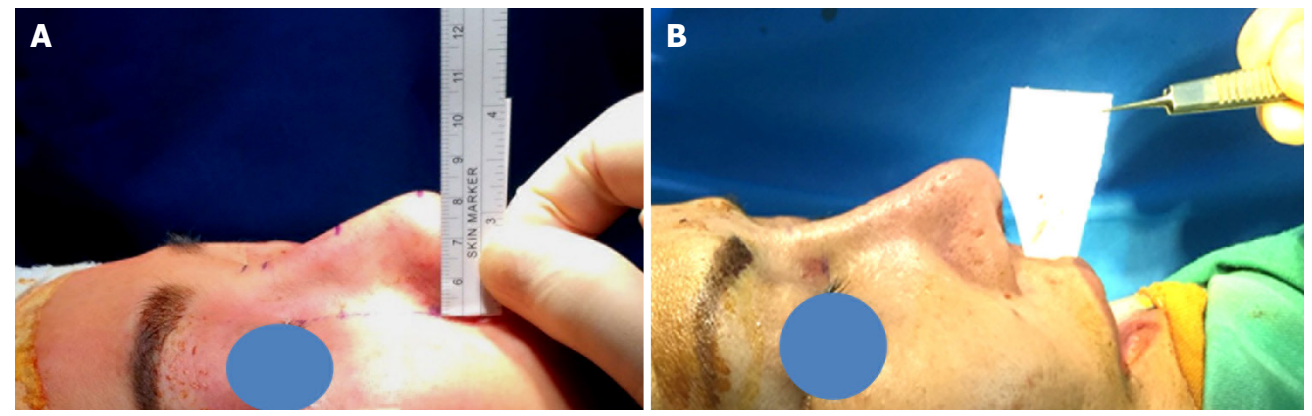

Figure 4. A: Intraoperative measurement of profile heights at nasion, bridge at mid-pupil level, rhinion and nasal tip; B: a paper is cut to check the nasolabial angle before and after surgery

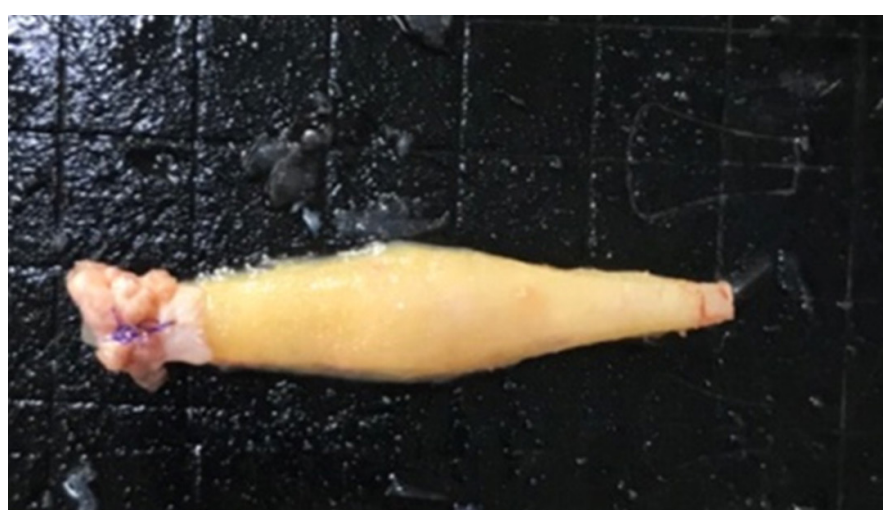

Figure 5. Fusiform shaped carved rib cartilage, perichondrium is placed on the cephalic end of the onlay graft to smoothen the radix contour

\section{INTRAOPERATIVE MEASUREMENT}

Before we carve the rib, we measure the height of the nose at 4 points in order to monitor the profile change during the operation [Figure 4] and check the contour change: (1) nasion height from the medial canthus; (2) bridge height at mid-pupil level; (3) rhinion; (4) nasal tip.

The shape we carve the rib graft into can be best described as a "fusiform" shape. It is tapered off on both ends and has a wider mid region [Figures 5-9]. We use the longer portion of the harvested costal cartilage for dorsal augmentation and the remaining shorter portion for other grafts (splint grafts, lateral crura strut 


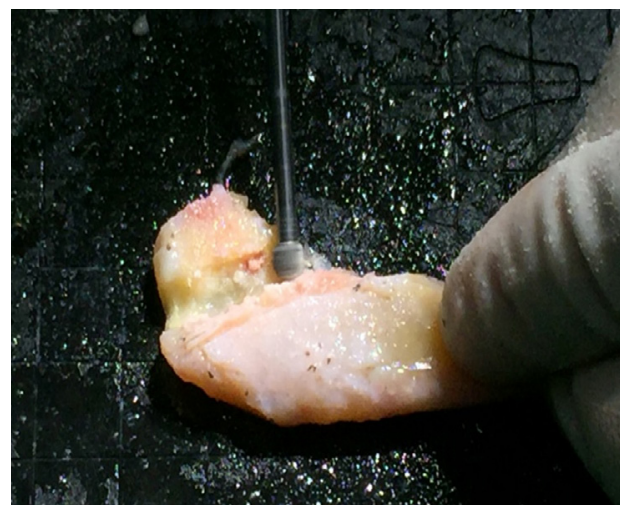

Figure 6. Powered instrument with cutting burr is used to carve the costal cartilage if it is calcified

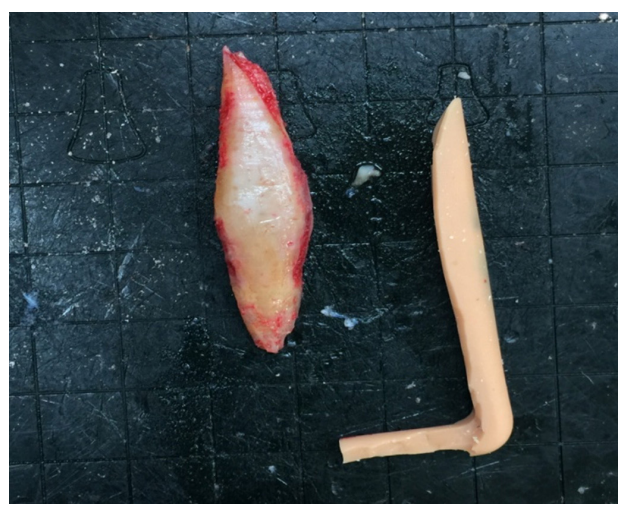

Figure 7. Partially calcified costal cartilage is sculptured according to the silicone implant

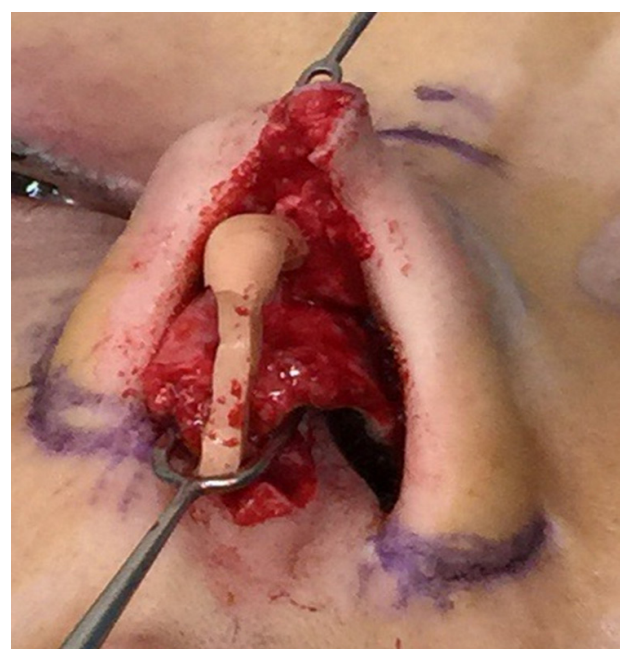

Figure 8. Intra-operatively, an L-shaped silicone implant is seen after opening the fibrotic capsule

grafts, caudal septal extension graft). The posterior aspect of the rib lies over the nasal area (over the nasal bones and cartilaginous dorsum) and is carved to blend with the underlying nasal framework. Asians have a rather low radix height. To ameliorate this, we carve the superior aspect of the graft in an uphill-converging manner, which rests effortlessly over the underlying bone, forming a new, enhanced radix. The undersurface of the graft is carved with a No. 10 blade in an inverted, flattened out " $U$ " fashion, along the natural contour 


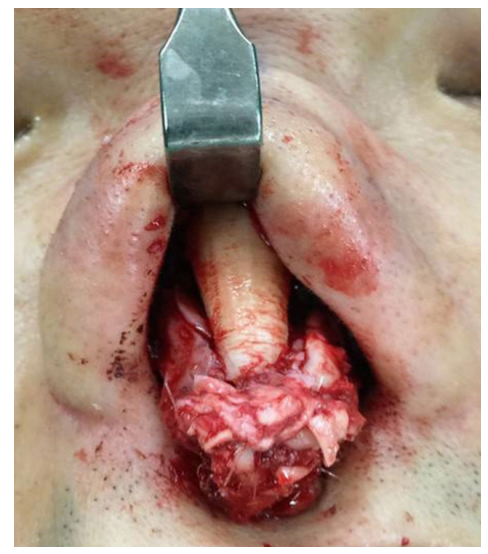

Figure 9. Intraoperative picture showing graft placement for dorsal augmentation
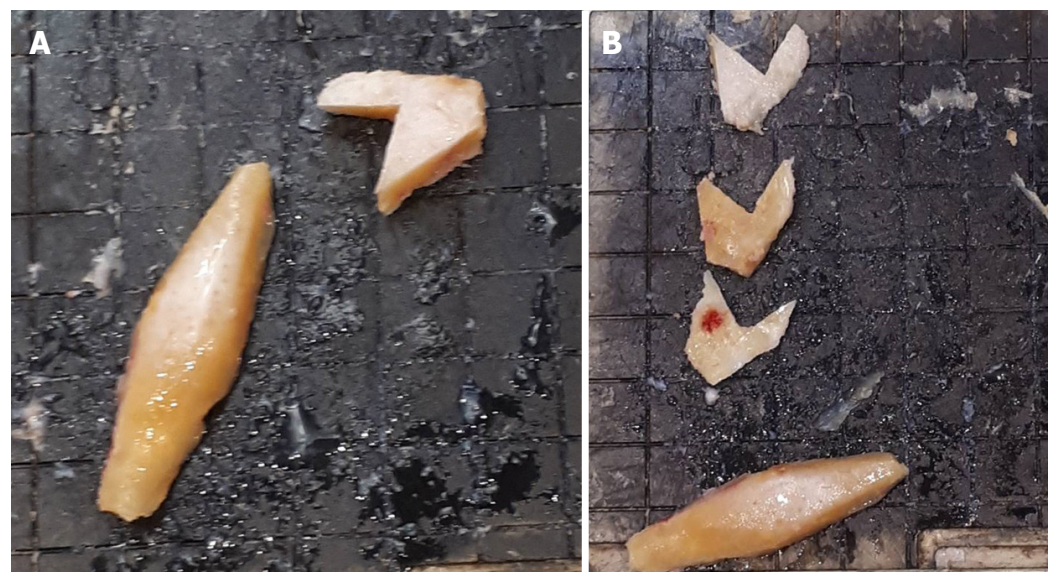

Figure 10. A: Medial portion of harvested rib cartilage carved into a dorsal onlay graft and the remaining lateral portion; B: the lateral portion of the harvested rib is split into three pieces which are used as caudal septal extension graft and splint grafts

of the harvested rib, for better fixation of the onlay graft over the underlying nasal framework. If the patient requires or wishes for a higher dorsum, we can assemble an additional rib cartilage underneath the onlay graft, carved to mingle with the underlying nasal framework and raise the overlying harvested rib for dorsal augmentation. Carving is done carefully to taper the margins of the rib so as not to make the graft too obvious or visible postoperatively. The caudal end of the rib graft is narrowed which harmonizes with the nasal bridge. While carving, we make sure that the end result is not a very narrow looking nose, nor is it too broad. We always keep in mind that a graft that is too narrow might not be in tone with the rest of the Asian facial features. We seldom use diced cartilage for dorsal augmentation as its absorption rate is very unpredictable and the nasal skin surface may appear irregular.

Splint grafts are carved either from the remaining septum or the harvested rib. The lateral or shorter part of the L-shaped harvested rib, is split into three identical grafts. The grafts are sliced in a longitudinal direction. The central part of the sliced rib is generally used as a caudal septal extension graft (CSEG) to minimize warping. The CSEG is carved into an approximately $2 \mathrm{~mm}$ thick graft. The length and height of the rib graft to be carved into a CSEG can be manipulated according to the requirements of the patient. The peripheral sliced grafts are further carved into spreader grafts or splint grafts [Figure 10]. The splint grafts are shaped as a trapezoid - the key point is that they need to support the septal cartilage along with the CSEG and form a strong platform. The remaining cartilage from the medial rib, after carving out the required amount needed 


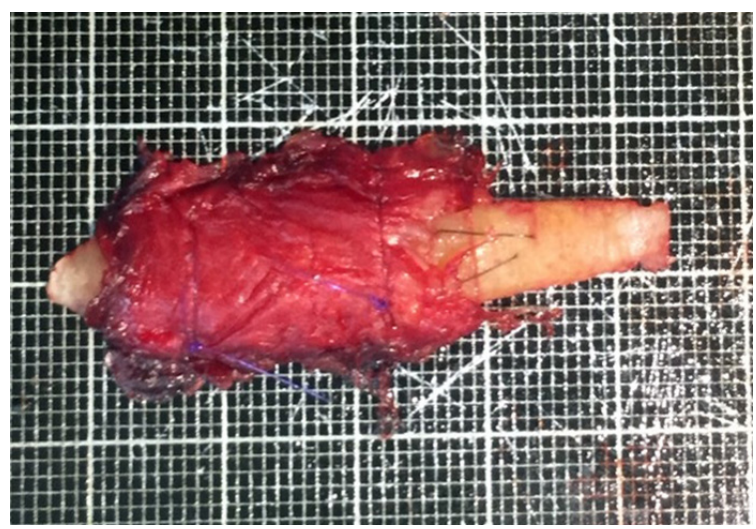

Figure 11. Rib graft covered with deep temporal fascia on the convexity

for the dorsal augmentation, can be carved into spreader grafts. We use spreader grafts only when the patient undergoes extensive osteotomy, if there is evidence of internal valve collapse or if there is deviation of the cartilaginous dorsum.

Owing to its curvature and elasticity, we prefer using conchal cartilage as lateral crura strut grafts (LCSG). If conchal cartilage is deficient or if we decide to use the rib as a LCSG, a small part of the rib is carved into a rectangular graft tapered on both sides.

\section{Graft placements and osteotomy}

After placing the rib onlay graft, we secure it in position by applying two or three fixation sutures with 5.0 PDS around the graft and through bilateral upper lateral cartilages (ULC). If a capsule is present due to a previous silicone implant, it is preserved and wrapped gently around the rib cartilage graft to minimize irregularity and thinning of the skin soft tissue envelope. Perichondrium of costal cartilage or deep temporal fascia can also be harvested and wrapped around the rib graft to hide any irregularities [Figure 11].

The caudal septal extension graft is fashioned from the septal cartilage or the rib graft in a trapezoidal shape. We place it in the midline, between bilateral medial crura and fixed to the caudal septum in an endto-end fashion or overlapped over the caudal end of the septum (depending on the strength and resilience of the remaining septal cartilage). End-to-end fixation of CSEG can prevent deviation. Splint grafts are used on either side over the dorsal septum to secure the CSEG at the midline and the lower end of CSEG is fixed near the anterior nasal spine (ANS). We are careful not to fix it too close to the ANS, to avoid any postoperative discomfort, columellar tilt or upper philtrum crease, which may be apparent on smiling and could be cosmetically unappealing. It is also paramount for bilateral medial LLC to be sutured in symmetry to prevent tip deformity.

According to the desired nasal shape and skin thickness, we insert other grafts such as lateral crura struts, batten grafts or tip-shield grafts. We rarely perform osteotomies on Asian patients because augmentation itself can improve a broad bridge. If the patient has a crooked bony dorsum, we perform intranasal medial and lateral osteotomies. Medial osteotomy begins at the junction of ULC and nasal bone at a paramedian position, preserving optimal width of the bony dorsum to prevent inverted $\mathrm{V}$ deformity or other deformities. We curve the cut of the medial osteotomy gently outwards (approximately $10^{\circ}-15^{\circ}$ ) as we proceed upwards, ensuring that osteotomy is complete and does not move too far cephalically into the frontal bone. It is then connected with the lateral osteotomy. This is how we avoid "rocker" deformity. We use the low-lowhigh fashion for lateral osteotomy as opposed to the high-low-high osteotomy performed in Caucasians. To circumvent narrowing of the nasal valve area, we start the lateral osteotomy at the level of the inferior 
turbinate. This can avoid step-like deformity, nasal block or collapse with preservation of periosteum along the osteotomy route.

Other steps such as alar flare reduction and rim strut grafts are performed as needed. We make sure to preserve as much tissue as possible to avoid scarring (which could be a result of multiple rhinoplasties).

\section{DISCUSSION}

Rib grafting has several advantages in revision rhinoplasty, especially for complications from filler injection rhinoplasty or artificial nasal implantation, which is very common in Asian patients. It offers an abundant supply of cartilage for use and rigid support. The chances of infection, skin necrosis and shrinkage are

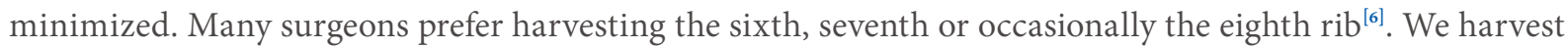
the sixth rib cartilage in females because the oblique incision scar can usually be hidden over the inframammary fold, and the seventh rib cartilage in males because the seventh rib cartilage is usually the longest one. If the patient is older than 40 years, a CT scan of chest wall may be needed to evaluate calcification of cartilage.

Complications such as warping can be overcome by balanced carving and allowing 15 min for maximal warping to occur ${ }^{[7]}$. We harvest the rib perichondrium as it can serve as an extra graft material. It can be used to camouflage skin thinning of the nasal tip and can also be used over the rib onlay graft to minimize noticeable graft contour. The incision for harvesting a rib graft may vary from 1.0-5 cm, depending on the patient's anterior chest wall thickness and surgeon's skill. Rib carving requires a lot of experience and versatility on the surgeon's part. The edges of the dorsal onlay graft may show irregularities even after fine trimming. Infection is not uncommon in secondary rhinoplasty. Meticulous dissection during surgery, effective antibiotics and appropriate postoperative care are very important. Cigarette is absolutely prohibited at least two weeks before and 1-2 months after surgery. The pre and post operative pictures of a few of our cases are listed below [Figures 12 and 13]. In cases of wound infection, the patients are treated with wound debridement, removal of inflamed cartilage and intravenous antibiotics. The reasons for infection could be the mass effect of harvested graft that affect the skin tension and disturb the nutrient/waste exchange diffusion process and also due to the poor blood supply in the recipient area in revision cases ${ }^{[7]}$.

To obtain aesthetically pleasing results, ensure patient satisfaction and minimize complications, the rhinoplasty surgeon must possess a thorough knowledge of nasal anatomy and ideal facial aesthetic proportions ${ }^{[8]}$. Many Asian patients undergo more than three revision surgeries to correct or offset improperly performed surgeries, the improper use of implants or surgical complications ${ }^{[9]}$. In a study by Park and Jin ${ }^{[10]}, 47$ percent were revision cases of rhinoplasty and four of five infection cases occured in their revision operations.

The current best evidence for techniques and complication rates in costal cartilage associated with rhinoplasty are based on case series ${ }^{[1]}$. Hence, complication rates depend on the surgeon's surgical skills as well as the patient's compliance.

In conclusion, revision rhinoplasty in Asians is a challenging surgery. Most of the revision cases require previous graft removal or reshaping, harvesting of a new graft, creating a clean plane and facing the scar tissues formed by previous surgeries. When a large amount of grafting is required, the costal cartilage can provide an ample amount of cartilage graft material. As the possibilities of complications should also be anticipated in revision rhinoplasty, rhinoplasty surgeons need more experience and learning from follow-up patients. 

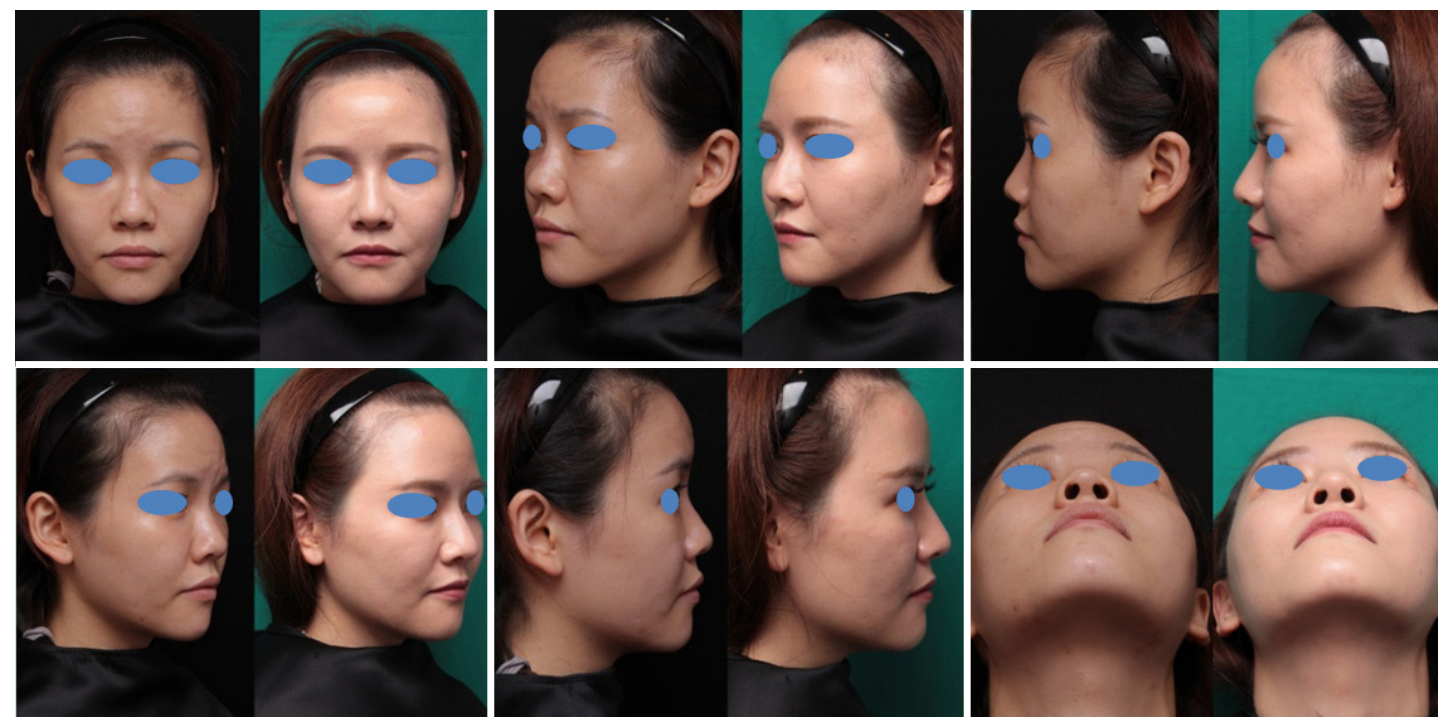

Figure 12. A 25-year-old female had filler injectable rhinoplasty thrice previously, as well as threads insertion. She underwent open rhinoplasty with autogenous costal cartilage grafting and ear cartilage grafting for tip refinement. These pictures show before and one year after surgery

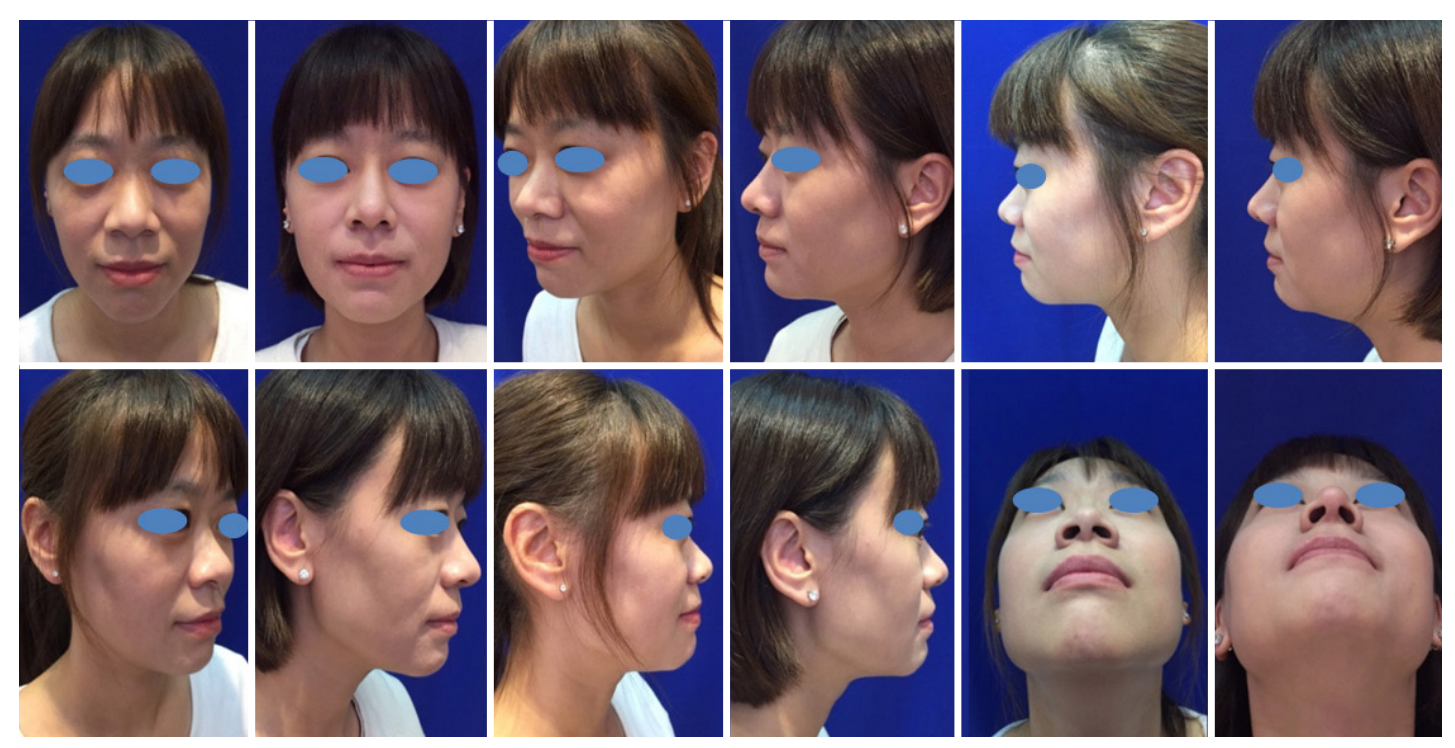

Figure 13. This female underwent open rhinoplasty with autogenous costal cartilage grafting and ear cartilage grafting for tip refinement. These pictures show before and three months after surgery

\section{DECLARATIONS}

\section{Acknowledgments}

We wish to express our thanks to The Ministry of Health and Welfare, Republic of China, for supporting the study program for Dr. Sarina Rajbhandari from Nepal, through the "Global Surgery Seed Training Program" atTri-Service General Hospital, Taipei, Taiwan.

\section{Authors' contributions}

Made contribution to the conception and design of the study and provided cases and material for the study: $\mathrm{Kao} \mathrm{CH}$

Made contribution to the presentation of the text and concept of the study: Rajbhandari S 


\section{Availability of data and materials}

Not applicable.

\section{Financial support and sponsership}

None.

\section{Conflicts of interest}

Both authors declared that there are no conflicts of interest.

\section{Ethical approval and consent to participate}

Written informed consent was obtained.

\section{Consent for publication}

Written informed consent was obtained for all patient images.

\section{Copyright}

(c) The Author(s) 2019.

\section{REFERENCES}

1. Marin VP, Landecker A, Gunter JP. Harvesting rib cartilage grafts for secondary rhinoplasty. Plast Reconstr Surg 2008;121:1442-8.

2. Lee MJ, Song HM. Asian rhinoplasty with rib cartilage. Semin Plast Surg 2015;29:262-8.

3. Kao CH, Hsu CH, Lee JC, Wang HW, Tsai KK. Customized material choice for Asian rhinoplasty: how we do it. Clin Otolaryngol 2011;36:165-70.

4. Bussi M, Palonta F, Toma S. Grafting in revision rhinoplasty. Acta Otorhinolaryngol Ital 2013;33:183-9.

5. Toriumi DM, Pero CD. Asian rhinoplasty. Clin Plast Surg 2010;37:335-52.

6. Gunter JP, Cochran CS. Dorsal augmentation with autogenous rib cartilage. Semin Plast Surg 2008;22:74-89.

7. Moon BJ, Lee HJ, KJang YJ. Outcome following rhinoplasty using autologous costal cartilage. Arch Facial Plast Surg 2012;14:175-80.

8. Lagura EMA, Yap EC, Garcia AVG. Augmentation rhinoplasty with rib cartilage graft. Philipp J Otolaryngol Head Neck Surg 2015;30:29-33.

9. Suh MK, Ahn ES, Kim HR, Dhong ES. A 2-year follow-up of irradiated homologous costal cartilage used as a septal extension graft for the correction of contracted nose in Asians. Ann Plast Surg 2013;71:45-9.

10. Park JH, Jin HR. Use of autologous costal cartilage in Asian rhinoplasty. Plast Reconstr Surg 2012;130:1338-48.

11. Varadharajan K, Sethkumar P, Anwar M, Patel K. Complications associated with the use of autologous costal cartilage in rhinoplasty: a systematic review. Aesthet Surg J 2015;35:644-52. 